\title{
Femtosecond vibrational transition-state dynamics in a chemical reaction
}

\author{
S. Pedersen, L. Bañares, a) and A. H. Zewail

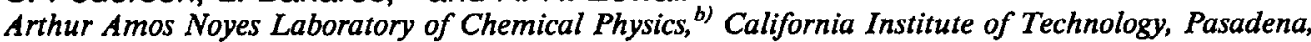 \\ California 91125
}

(Received 27 August 1992; accepted 30 September 1992)

When excited directly, complexes in transition states undergo nuclear motions characteristic of bound, quasibound, or unbound dynamics. In elementary reactions, the motions are on the femtosecond time scale and depend on the dimensionality of the potential. ${ }^{1}$ For example, in the reaction $A B A^{* \dagger} \rightarrow A+B A$, there are two relevant coordinates that describe the motion of the triatomic transition state species, $\mathrm{ABA}^{*+}$ : the symmetric and antisymmetric stretch modes, in addition to the bend. Near the saddle point, the motion is bound in the symmetric stretch and unbound in the antisymmetric stretch (reaction coordinate), and the complex could exhibit vibrational motion in the symmetric coordinate, perpendicular to the reaction coordinate. This quantized and bound motion is expected to remain at infinity (i.e., final products) with well defined coherence, as observed experimentally ${ }^{1}$ and studied theoretically. ${ }^{1,2}$

The behavior perpendicular to the reaction coordinate is different from the Feshbach-type resonance along the reaction coordinate. For this type of resonance, popular in reactive scattering (cross section vs energy), the motion is quasibound along the reaction coordinate and the complex is trapped (finite lifetime), but has no bound motion at infinity. ${ }^{3}$ Transition state (TS) vibrational dynamics, which are important to the understanding of the nature of the potential in the TS region, ${ }^{4-7}$ are particularly relevant to studies which directly or indirectly probe the TS. These include emission ${ }^{4}$ and Raman scattering during dissociation, ${ }^{5}$ temporal dynamics, ${ }^{1}$ photodetachment spectra, ${ }^{6}$ peaks in the continuum spectra of dissociation reactions, ${ }^{8}$ and structure (steps) in the rate constants vs energy. ${ }^{9}$

For systems with a large number of degrees of freedom $(N)$, the situation is more complex. First, the one coordinate, perpendicular to the reaction coordinate, is now replaced by the $N-1$ of TS theory. ${ }^{10}$ Despite the large $N$, quantum localization may occur on a dynamic potential. ${ }^{11}$ Second, the wave packet motion may suffer fast spreading as its structure is now made of a large number of modes. The isomerization of diphenylethylene (stilbene) is an example of such a complex reaction with 72 modes; the reaction coordinate is described by a single motion about the double bond (torsional angle $\theta$ ). ${ }^{12}$ The molecule at the $c i s$ configuration is unbound (or quasibound) in the $\theta$ coordinate, but, in principle, is bound along all the other coordinates: a saddle point TS in its strict definition.

In this communication, we report the observation of the femtosecond dynamics of the TS in the isomerization reaction of cis-stilbene under collisionless conditions. An isolated coherent motion that lasts for several hundred femtoseconds and depends on the total excess vibrational energy has been observed. Such motion correlates with the topology of the potential energy surface (PES) and represents a nonstatistical behavior. The experimental results are compared with calculations on a model PES for the reaction.

As shown in Fig. 1, the wave packet is initially prepared by a UV femtosecond laser pulse. The temporal evolution of the wave packet is then probed by resonance MPI in a pump-probe scheme. ${ }^{13}$ As in trans-stilbene, the ionization efficiency ${ }^{14}$ of $c i s$-stilbene is high due to the existence of resonant intermediate states, studied theoretically ${ }^{15}$ and experimentally. ${ }^{16}$ The PES in Fig. 1 illustrates the methodology.

The experimental apparatus has been described in detail elsewhere. ${ }^{17}$ Here, the two beams (pump and probe pulses, $\sim 80 \mathrm{fs}$ ) were collinearly recombined and focused into the ionization chamber, evacuated to $\sim 10^{-5}$ Torr. The cis-stilbene used in these experiments was of very high purity $(99.98 \%)$, courtesy of the Saltiel group who have used it in careful studies in solutions. ${ }^{18}$

Figure 2(b) shows the measured transient for $\lambda_{\text {pump }}$ $=306 \mathrm{~nm}, \lambda_{\text {probe }}=2 \times 612 \mathrm{~nm}$. The decay curve is nonexponential and shows a peak at a time delay of $\sim 0.5 \mathrm{ps}$. The transient has an exponential decay (lifetime) and the oscillatory pattern in Fig. 2(a) [see Eq. (1)]. The singleexponential fit was obtained using a nonlinear least-squares method where the finite and measured response function was included. We have used the following molecular response function to describe the population and coherence decays: ${ }^{19}$

$$
M(t)=\exp (-\gamma t) \cdot\{a+b \cos [\omega(t) \cdot t]\},
$$

where $\gamma$ is the decay rate and $a, b$ are constants. The timedependent frequency is $\omega(t)=2 \pi /\left(T_{0}+k t\right)$, where $T_{0}$ is the initial period (Fig. 1) and $k$ gives its change with time. The single-exponential fit of the transient gives $\gamma^{-1}=307 \mathrm{fs}$ and $a=1.37$. For the data in Fig. 2(a), $a=0$, and we obtained $b=0.25, T_{0}=360 \mathrm{fs}$, and $k=0.42$. The oscillatory bchavior was found to be independent of the relative polarization ${ }^{20}$ and intensity (linear regime) of the pump and probe pulses.

Greene and Farrow ${ }^{22}$ were the first to measure the initial decay of $c i s$-stilbene in the gas phase. They reported a single exponential decay with a long lifetime component attributed to some trans impurity. As discussed above, the decay is observed to be nonexponential with a peak structure. This behavior is lost when wider pulses are used. In their case, the decay was reported to be $320 \pm 20 \mathrm{fs}$ and the 

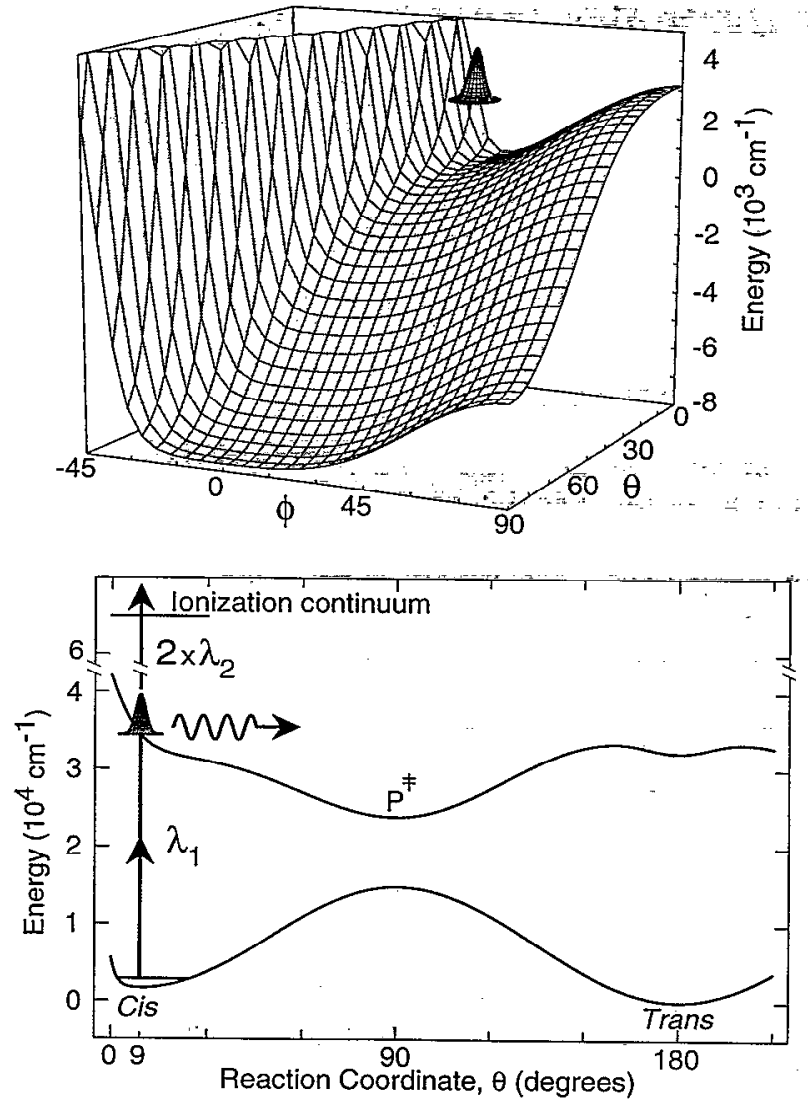

FIG. 1. The potential energy surface describing the isomerization reaction along the $\theta$ and $\phi$ coordinates, maintaining all other degrees of freedom fixed (see text). The cut at the bottom showing the potential energy vs $\theta$ represents the twisting about the double bond. $P^{\dagger}$ is the perpendicular state with minimum energy along $\theta$. The initial preparation of the wave packet is by $\lambda_{1}$ and the probing is by $2 \times \lambda_{2}$ (through ionization). Note that the zero of energy is different in the two figures. The initial wave packet is at a nonequilibrium configuration, as determined by $\lambda_{1}$.

laser pulse widths were $250 \mathrm{fs}$. Here, our pulses are shorter by a factor of $\sim 3$, and the peak is clearly visible. Also, there is no long decay component (trans impurity) in our transients. Figure 2(b) shows the same set of data, but now for a higher pump energy; note the shape near the $t=0$ region. The total vibrational energy was changed from $\sim 4300 \mathrm{~cm}^{-1}$ (at $306 \mathrm{~nm}$ ) to $\sim 6700 \mathrm{~cm}^{-1}$ (at $285 \mathrm{~nm}$ ). ${ }^{23}$

In the isomerization region there are at least three angular coordinates, out of the 72 modes, directly involved: the $C_{e}-C_{e}$ torsional angle, $\theta$ (reaction coordinate), the $C_{e}-$ $C_{e}-C_{P h}$ in-plane bending angle, $\alpha$, and the $C_{e}-C_{P h}$ torsional angle, $\phi$ (Fig. 3). Myers and Mathies ${ }^{28}$ estimated that the torsional angle $\theta$ increases from $9^{\circ}$ to $34^{\circ}$ during the first 20 fs (vide infra).

Petek et $a l^{25,29}$ have addressed the issue of the dimensionality of the PES. They concluded that the $\phi$ and $\alpha$ coordinates must be considered not only in the isomerization but also in the photocyclation. ${ }^{30}$ We have modified the Frederick et al. $\mathrm{PES}^{29}$ to include a complete $\theta$ dependence $^{33}$ for the system to reach large $\theta$ 's, up to $\pi / 2$ and $\pi$ (Fig. 1). To obtain the dynamics we have considered the wave packet centered at the equilibrium configuration $(\theta$
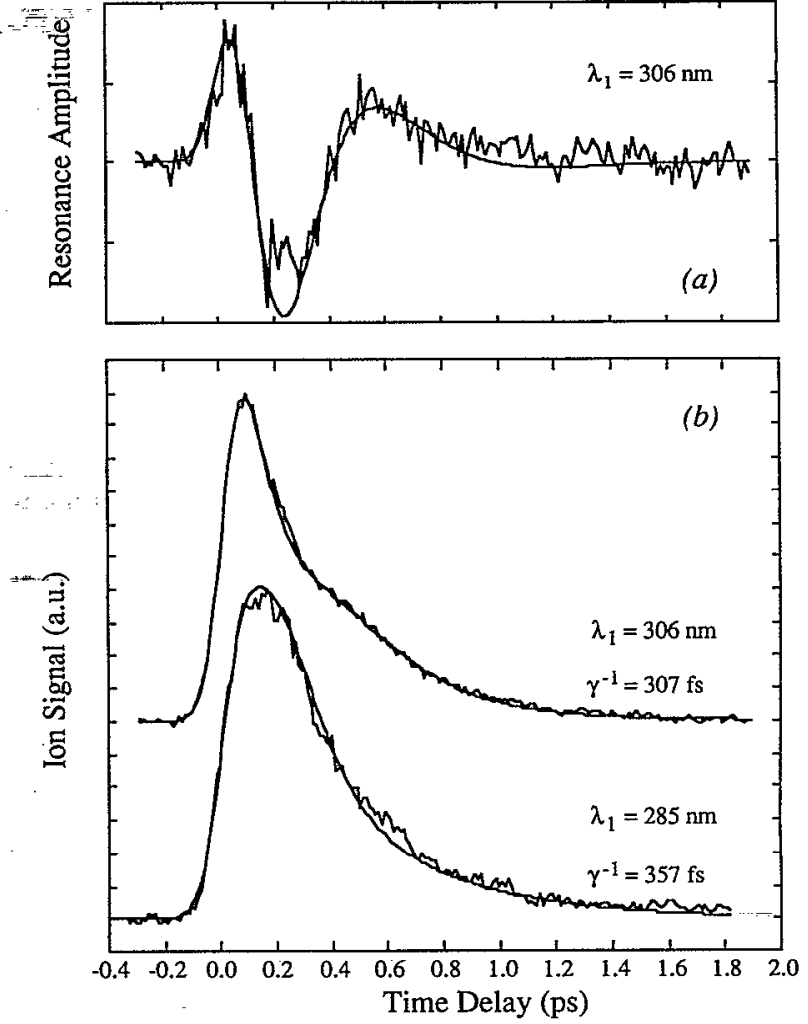

FIG. 2. Femtosecond transients obtained from the $\lambda_{1}=306 \mathrm{~nm}$ and 285 $\mathrm{nm}$ experiments. (a) The vibrational resonance [cosine term of (1)] amplitude for $\lambda_{1}=306 \mathrm{~nm}$ was obtained from (b) after subtracting the overall decay component of Eq. (1) (see text). The smooth line is the best fit using Eq. (1) (setting $a=0$ ) with the parameters listed in the text. The same parameters (with $a=1.37$ ) reproduce the solid line fit in (b). Also shown in (b) is the femtosecond transient obtained from the $\lambda_{1}=285 \mathrm{~nm}$ experiments. The smooth line is the result of the fit using Eq. (1) and similar methodology to the $\lambda_{1}=306 \mathrm{~nm}$ data treatment. A single exponential does not fit the data well, particularly at early times.

$=9^{\circ}, \phi=26.8^{\circ}, \alpha=124^{\circ}$ ) at $t=0$. The time evolution in each coordinate was obtained by solving the equation of motion for $\dot{\theta}(t), \dot{\alpha}(t)$, or $\dot{\phi}(t)$, knowing the total energy and the derived PES.

At the total energy of interest $\sim 2600 \mathrm{~cm}^{-1}$ (vide infra), the wave packet motion in the $\theta$ coordinate takes $\sim 210$ fs to end at $\theta=90^{\circ}$. Along $\phi$, half the vibrational period is $\sim 340 \mathrm{fs}$, comparable to the decay time along $\theta$. According to the results of Fig. 2, the wave packet displays not only a decay but also a significant spreading and a change of the period. This is consistent with the motion being along the $\theta$ valley which "opens up" with time; the period changes by $k x$ time. The changes in the PES are shown in Fig. 3, together with the representation of the correlated motions at different $\theta$ 's.

There are several points to be made here. First, the absorption spectrum of cis-stilbene is unstructured, ${ }^{34}$ and the results reported here indicate that this simply reflects the steep slope of the potential in the Franck-Condon region; there are two time scales involved: one for the initial dephasing and the other for the nuclear dynamics. ${ }^{1,5,35} \mathrm{Sec}-$ ond, the localization of motion is consistent with a Born- 

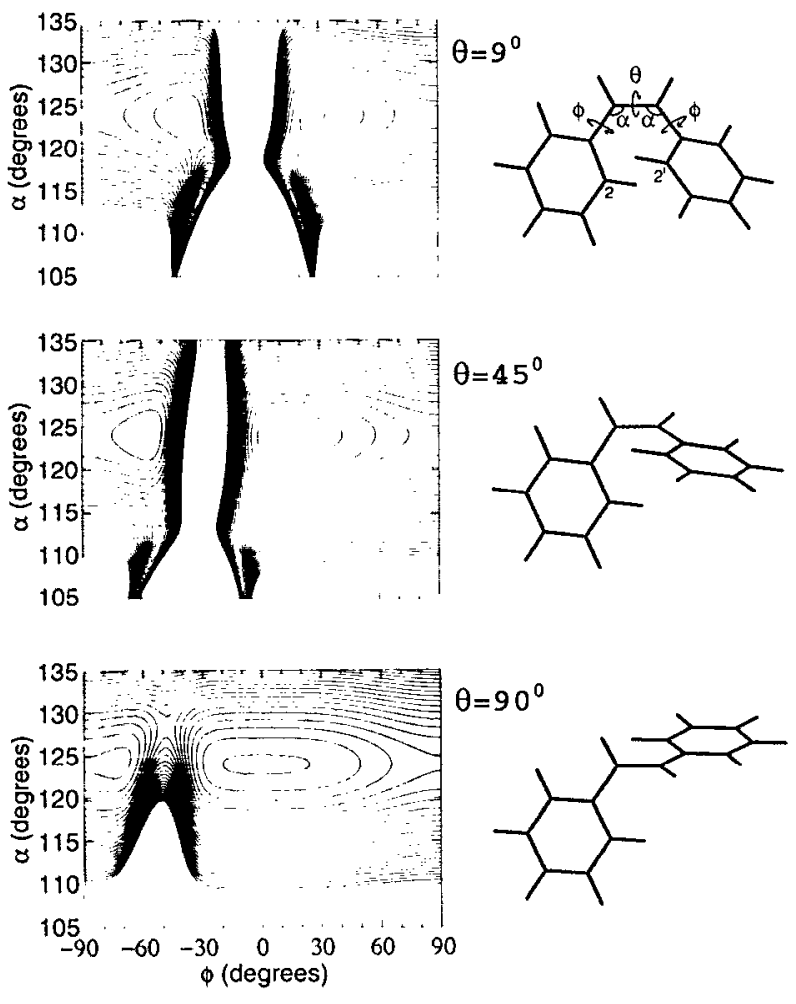

FIG. 3. Calculated PES for the cis-stilbene system as outlined in the text. The angles of interest are defined in one of the structures. Notice that at low $\theta$ there are two minima in the $\alpha-\phi$ surface corresponding to cisstilbene and DHP separated by a barrier, of height $\sim 535 \mathrm{~cm}^{-1}$, above the cis minimum (see Ref. 29). As $\theta$ is increased, the DHP configuration becomes more unstable and the stilbene well more stable and wider. At $\theta=90^{\circ}$, the only stable configuration is twisted stilbene (phantom state). We made the $\theta$ dependence to include the change in the potential energy given in the text and in Fig. 1. This change in $\theta$ is a modification of the PES in Ref. 29. The contour lines in all three plots are separated by 1000 $\mathrm{cm}^{-1}$. The lowest and highest contour lines for the different plots are (a) $\theta=9^{\circ},-8600 \mathrm{~cm}^{-1}$, and $15400 \mathrm{~cm}^{-1}$, (b) $\theta=45^{\circ},-1600 \mathrm{~cm}^{-1}$, and $22400 \mathrm{~cm}^{-1}$; and (c) $\theta=90^{\circ},-7600 \mathrm{~cm}^{-1}$, and $16400 \mathrm{~cm}^{-1}$

Oppenheimer dynamical separation ${ }^{11}$ of vibrational frequencies.

Third, the deduction of the slope of the potential at the Franck-Condon region, obtained from resonance Raman studies, ${ }^{28}$ is consistent with the time scale that we have calculated for the $\theta$ motion. However, our calculated time is much longer than that deduced using the approximation made in Ref. 28 for relating $\theta$ to time. ${ }^{36}$ If we consider the $\theta$ motion, the time scale depends on how far the system is twisted. ${ }^{37}$ For example, from $\theta=9^{\circ}$ to $20^{\circ}$ and back to $9^{\circ}$, the motion takes only $\sim 80 \mathrm{fs}$ (energy, $\sim 2600 \mathrm{~cm}^{-1}$ ), much shorter than the resonance period. But if the packet moves on beyond $\theta=20^{\circ}$, there is a well at the phantom configuration $\left(\theta=90^{\circ}\right)$ and IVR to other available modes will produce a very long "return time," back to the $\theta<20^{\circ}$ region. At $\theta=90^{\circ}$, if the energy is lowered (by IVR) below that of the cis configuration, the wave packet becomes bound. We have assumed that $\sim 60 \%$ of the total energy goes to the mode of interest, but if more energy is allocated to other modes, slower dynamics will be observed. The fraction of energy in each mode is not known without further experiments.
As the total energy was increased ( $285 \mathrm{~nm}$ excitation), the results in Fig. 2(b) were observed: the decay gets longer and near the $t=0$ region the transient does not fit a single exponential. At this energy, the motion is above the barrier in the $\phi$ coordinate (free phenyl rotation), and we expect a significant spreading of the wave packet. Moreover, bath modes may exchange energy with the relevant isomerization modes and this may result in a nonexponential behavior, in analogy with "diffusive" motions. The overall decay of the wave packet is slower than that at lower excess energy, indicating a nonstatistical behavior. In view of the time scales involved, this reaction represents a case where there is not enough time for conventional $\mathrm{IVR}^{38}$ and it is not clear that TS theory is appropriate for rate constants.

There are further studies to be made and these include: (a) calculation of the dynamics on the multidimensional PES, and including the bath modes; ${ }^{40}$ (b) studies at lower internal energy in supersonic beams; and (c) identification of the Franck-Condon modes and their fractional energies. In our full account ${ }^{41}$ we plan to complete these studies for this and related reactions.

Note added in proof. We have recently obtained the fs transients in a molecular beam with helium and argon as the expanding gases. The isolated molecules and the cluster dynamics are currently under examination. In a recent paper by the Hochstrasser group [J. Chem. Phys. 97, 5239 (1992)], they concluded that complete IVR in solution is not observed. Troe's group [J. Chem. Phys. 97, 4827 (1992)] has shown, from temperature and viscosity dependences, the need of a bottleneck in explaining the dynamics in solutions. The nonstatistical behavior reported here should be relevant to these new studies in solutions.

We wish to thank Professor J. Saltiel for helpful discussions and for providing us with the ultrapure sample. We also wish to acknowledge stimulating discussions with Professor W. Miller, Professor R. Marcus, and Professor D. Neumark. This work was supported by a grant from the US AFOSR and by the NSF.

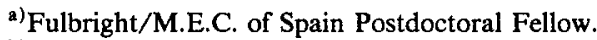

b) Contribution No. 8712 .

${ }^{1}$ For a recent review, see A. H. Zewail, Faraday Discuss. Chem. Soc. 91, 207 (1991), and references therein.

${ }^{2}$ For a recent study, see J. Manz, B. Reischl, T. Schröder, F. Seyl, and B. Warmuth, Chem. Phys. Lett. (in press, 1992).

${ }^{3}$ (a) See the papers in the recent volume Faraday Discuss. Chem. Soc. 91, (1991); (b) W. H. Miller, Annu. Rev. Phys. Chem. 41, 245 (1991).

${ }^{4}$ H.-J. Foth, J. C. Polanyi, and H. H. Telle, J. Phys. Chem. 86, 5027 (1982).

${ }^{5}$ D. Imre, J. L. Kinsey, A. Sinha, and J. Krenos, J. Phys. Chem. 88, 3956 (1984).

${ }^{6}$ D. M. Neumark, Annu. Rev. Phys. Chem. 43, (1992). See, also, the author's papers in Ref. 3.

${ }^{7}$ (a) P. R. Brooks, Chem. Rev. 88, 407 (1988); (b) M. D. Barnes, P. R. Brooks, R. F. Curl, and P. W. Harland, J. Chem. Phys. 94, 5245 (1991); (c) M. D. Barnes, P. R. Brooks, R. F. Curl, P. W. Harland, and B. R. Johnson, ibid. 96, 3559 (1992).

${ }^{8}$ See, for example, (a) C. Jouvet, M. Boivineau, M. C. Duval, and B. Soep, J. Phys. Chem. 91, 5416 (1987); W. H. Breckenridge, C. Jouvet, and B. Soep, J. Chem. Phys. 84, 1443 (1986); (b) P. D. Kleiber, A. M. Lyyra, K. M. Sando, S. P. Heneghan, and W. C. Stwalley, Phys. Rev. 
Lett. 54, 2003 (1985); (c) L. J. Butler, Chem. Phys. Lett. 182, 393 (1991).

${ }^{9}$ (a) E. R. Lovejoy, S. K. Kim, and C. B. Moore, Science 256, 1541 (1992); (b) G. A. Brucker, S. I. Ionov, Y. Chen, and C. Wittig, Chem. Phys. Lett. 194, 301 (1992); (c) L. R. Khundkar, J. L. Knee, and A. H. Zewail, J. Chem. Phys. 87, 77 (1987).

${ }^{10}$ See (a) R. A. Marcus, Science 256, 1523 (1992); (b) M. M. Kreevoy and D. G. Truhlar, in Investigations of Rates and Mechanism of Reactions, edited by C. F. Bernasconi (Wiley, New York, 1986), Vol. 6, Part 1, p. 13; (c) P. Pechukas, Annu. Rev. Phys. Chem. 32, 159 (1981); (d) See, also, Ref. 3.

${ }^{11}$ (a) G. Hose, H. S. Taylor, and Y. Y. Bai, J. Chem. Phys. 80, 4363 (1984); (b) B. Hartke, J. Manz, and J. Mathis, Chem. Phys. 139, 123 (1989).

${ }^{12}$ For reviews see (a) R. M. Hochstrasser, Pure Appl. Chem. 52, 2683 (1980); (b) J. Saltiel and Y.-P. Sun. Photochroism-Molecules and Systems, edited by $\mathrm{H}$. Durr and H. Bouas-Laurent (Elsevier, Amsterdam, 1990), p. 64.

${ }^{13}$ L. R. Khundkar and A. H. Zewail, Annu. Rev. Phys. Chem. 41, 15 (1990), and references therein.

${ }^{14}$ (a) J. W. Perry, N. F. Scherer, and A. H. Zewail, Chem. Phys. Lett. 103, 1 (1983); (b) N. F. Scherer, L. R. Khundkar, T. S. Rose, and A. H. Zewail, J. Phys. Chem. 91, 6478 (1987).

${ }^{15}$ (a) G. Orlandi, P. Palmieri, and G. Poggi, J. Am. Chem. Soc. 101, 3492 (1979); (b) G. Olbrich, Ber. Bunsenges. Phys. Chem. 86, 209 (1982); (c) G. Hohlneicher and B. Dick, J. Photochem. 27, 215 (1984).

${ }^{16}$ (a) B. I. Greene and T. W. Scott, Chem. Phys. Lett. 106, 399 (1984); (b) F. E. Doany, R. M. Hochstrasser, B. I. Greene, and R. R. Millard, ibid. 118, 1 (1985); (c) S. H. Courtney, S. K. Kim, S. Canonica, and G. R. Fleming, J. Chem. Soc., Faraday Trans. 2 82, 2065 (1986); (d) S. Abrash, S. Repinec, and R. M. Hochstrasser, J. Chem. Phys. 93, 1041 (1990); (e) J. K. Rice and A. P. Baronavski, J. Phys. Chem. 96, 3359 (1992).

${ }^{17}$ M. J. Rosker, M. Dantus, and A. H. Zewail, J. Chem. Phys. 89, 6113 (1988).

${ }^{18}$ J. Saltiel, A. Waller, Y.-P. Sun, and D. F. Sears, Jr., J. Am. Chem. Soc. 112, 4580 (1990).

${ }^{19}$ A. H. Zewail, M. Dantus, R. M. Bowman, and A. Mokhtari, J. Photochem. Photobiol. A Chem. 62, 301 (1992), and references therein.

${ }^{20}$ Measurements were made for parallel, perpendicular, and magic angle polarizations and the anisotropy was obtained as described elsewhere (Ref. 21). The oscillation was found to be the same in all polarizations and only the magnitude of the signal was affected.

${ }^{21}$ (a) P. M. Felker and A. H. Zewail, J. Chem. Phys. 86, 2460 (1987); (b) J. S. Baskin and A. H. Zewail, J. Phys. Chem. 93, 5701 (1989).

${ }^{22}$ B. I. Greene and R. C. Farrow, J. Chem. Phys. 78, 3336 (1983).

${ }^{23}$ The excess vibrational energies were calculated knowing the difference in energy between the trans and cis isomers in the ground $\left(1605 \mathrm{~cm}^{-1}\right)$ (Ref. 24) and excited (set at $\sim 500 \mathrm{~cm}^{-1}$ ) (Ref. 25) state. The thermal energy for cis-stilbene was calculated as described in a previous paper (Ref. 26) using the $S_{0}$ vibrational frequencies calculated by Warshel (Ref. 27). A thermal energy of $\sim 1740 \mathrm{~cm}^{-1}$ was obtained for a temperature of $25^{\circ} \mathrm{C}$.

${ }^{24}$ J. Saltiel, S. Ganapathy, and C. Werking, J. Phys. Chem. 91, 2755 (1987).
${ }^{25}$ H. Petek, Y. Yoshihara, Y. Fujiwara, Z. Lin, J. H. Penn, and J. H. Frederick, J. Phys. Chem. 94, 7539 (1990).

${ }^{26}$ J. L. Herek, S. Pedersen, L. Bañares, and A. H. Zewail, J. Chem. Phys. 98 (1992).

${ }^{27}$ A. Warshel, J. Chem. Phys. 62, 214 (1975).

${ }^{28}$ A. B. Myers and R. M. Mathies, J. Chem. Phys. 81, 1552 (1984).

${ }^{29}$ J. H. Frederick, Y. Fujiwara, J. II. Penn, K. Yoshihara, and H. Petek, J. Phys. Chem. 95, 2845 (1991).

${ }^{30}$ Photocyclation to dihydrophenanthrene (DHP) is another open channel for excited cis-stilbene. Former investigations in solution (Ref. 31) gave quantum yields of $35 \%$ and $10 \%$ for cis-to-trans and cis-to-DHP reactions, respectively. However, recently it was suggested that as much as $30 \%$ of excited cis-stilbene may undergo photocyclation to form excited DHP (Refs. 25 and 29). The dynamics toward DHP have been studied also in solution (Ref. 32) and ground-state DHP was found to be formed in $1.7 \mathrm{ps}$ in hexadecane.

${ }^{31}$ (a) K. A. Muszkat and E. Fischer, J. Chem. Soc. B 662, (1967); (b) T. Wismonski-Knittel, G. Fischer, and E. Fischer, J. Chem. Soc. Perkin Trans. 1, 1930 (1974); (c) S. Sharafy and K. A. Muszkat, J. Am. Chem. Soc. 93, 4119 (1971); (d) K. A. Muszkat, Top. Curr. Chem. 88, 89 (1980).

${ }^{32}$ S. T. Repinec, R. J. Sension, A. A. Szarka, and R. M. Hochstrasser, J. Phys. Chem. 95, 10380 (1991).

${ }^{33}$ The $\theta$ dependence has been empirically introduced by considering a truncated Fourier series in the torsional angle $\theta$ of the form $V_{\text {tor }}$ $=V_{2} \sin ^{2} \theta+V_{4} \sin ^{2} 2 \theta . V_{2}$ determines the height of the barrier to torsion about the double bond and has been taken to be $-7850 \mathrm{~cm}^{-1}$, as deduced from the slope of the potential estimated by Myers and Mathies (Ref. 28). $V_{4}$, which determines the flatness (shape) of the potential to torsion, was set at $1528 \mathrm{~cm}^{-1}$, maintaining the same $V_{2} / V_{4}$ ratio as for the conjugation potential of Ref. 29.

${ }^{34} \mathrm{H}$. Petek, Y. Fujiwara, D. Kim, and K. Yoshihara, J. Am. Chem. Soc. 110, 6269 (1988), and references therein.

${ }^{35}$ J. A. Beswick and J. Jortner, Chem. Phys. Lett. 168, 246 (1990).

${ }^{36}$ In Ref. 41 we will discuss these methods of calculation.

${ }^{37}$ The probe pulse can be in resonance on different parts of the PES, either along the reaction coordinate or perpendicular to it. In fact, one may assign the peak in Fig. 2 as a delayed probe along the $\theta$ coordinate with an induction period (Refs. 1 and 17). However, the resonance has a well defined phase change (cosine type), and also the time scale is not consistent with the potential characteristics as discussed in the text. As shown in Fig. 1, the probe is close to the the initial motion of the wave packet because of $\lambda_{1}$ promotion. Also, the twisting along $\theta$ takes the system away from resonance [Rer. 16(a)] due to large conformalional changes in $S_{1}$.

${ }^{38}$ At $\theta=180^{\circ}$, it is known that the trans form has a barrier of $3.3 \pm 0.2$ $\mathrm{kcal} / \mathrm{mol}$ to isomerization under collisionless conditions (Ref. 39). We have also studied the femtosecond dynamics of the trans configuration to compare with the cis. Unlike the cis, IVR is evident and we observe biexponential decay behavior which we shall detail in Ref. 41 .

${ }^{39}$ L. Banares, A. A. Heikal, and A. H. Zewail, J. Phys. Chem. 96, 4127 (1992), and references therein.

${ }^{40}$ (a) T. Carrington and W. H. Miller, J. Chem. Phys. 84, 4364 (1986); (b) S. Fischer and M. Karplus, Chem. Phys. Lett. 194, 252 (1992).

${ }^{41}$ L. Bañares, S. Pedersen, and A. H. Zewail (in preparation). 